Note

\title{
Development and Validation of an Event-Specific Quantitative PCR Method for Genetically Modified Maize MIR162
}

\author{
Reona Takabatake ${ }^{1}$, Tomoko Masubuchi ${ }^{1}$, Satoshi Futo ${ }^{2}$, Yasutaka Minegishi ${ }^{3}$, Akio Noguchi, \\ Kazunari Kondo ${ }^{4}$, Reiko Teshima ${ }^{4}$, Takeyo Kurashima ${ }^{1}$, Junichi Mano ${ }^{1}$ and Kazumi Kitta ${ }^{1, *}$ \\ ${ }^{1}$ Analytical Science Division, National Food Research Institute, \\ National Agriculture and Food Research Organization: \\ 2-1-12 Kannondai, Tsukuba, Ibaraki 305-8642, Japan; \\ ${ }^{2}$ FASMAC Co., Ltd.: \\ 5-1-3 Midorigaoka, Atsugi, Kanagawa 243-0041, Japan; \\ ${ }^{3}$ NIPPON GENE Co., Ltd.: \\ 1-8-7 Toiyamachi, Toyama, Toyama 930-0834, Japan; \\ ${ }^{4}$ National Institute of Health Sciences: \\ 1-18-1 Kamiyoga, Setagaya-ku, Tokyo 158-8501, Japan; \\ *Corresponding author
}

\begin{abstract}
A novel real-time PCR-based analytical method was developed for the event-specific quantification of a genetically modified (GM) maize event, MIR162. We first prepared a standard plasmid for MIR162 quantification. The conversion factor (Cf) required to calculate the genetically modified organism (GMO) amount was empirically determined for two real-time PCR instruments, the Applied Biosystems 7900HT (ABI7900) and the Applied Biosystems 7500 (ABI7500) for which the determined Cf values were 0.697 and 0.635 , respectively. To validate the developed method, a blind test was carried out in an interlaboratory study. The trueness and precision were evaluated as the bias and reproducibility of relative standard deviation (RSDR). The determined biases were less than $25 \%$ and the RSDR values were less than $20 \%$ at all evaluated concentrations. These results suggested that the limit of quantitation of the method was $0.5 \%$, and that the developed method would thus be suitable for practical analyses for the detection and quantification of MIR162.
\end{abstract}

(Received March 25, 2014)

Key words: MIR162; event-specific; genetically modified (GM); real-time PCR; maize

\section{Introduction}

The utilization of genetically modified (GM) crops has been increasing around the world ${ }^{1)}$. Many countries including Japan legislate labeling systems of authorized GM crops and/or their derived foods. To enforce the labeling system, it is indispensable to define a practical threshold level and validated quantitative methods for the estimation of unintentional commingling levels of authorized GM organisms (GMO). In Japan, the GMO labeling system is stipulated by the "Act on Standardization and Proper Quality Labeling of Agricultural Forestry Products (JAS Law)"*1 and the "Food Sanitation Act"*2, and the thresholds for approved GM maize and soybean events both have been set at $5 \%{ }^{2)}, * 3, * 4$. For GM

${ }^{* 1}$ Notification No. 9 (Aug. 31, 2011); Consumer Affairs Agency, Government of Japan (2011) http://www.caa.go.jp/jas/ hyoji/pdf/kijun_03.pdf

*2 Notification No. 370 (Aug. 31, 2011); Consumer Affairs Agency, Government of Japan (2011) http://www.caa.go.jp/ foods/pdf/syokuhin700.pdf maize, specific quantitative methods for five GM maize events (Bt11, Bt176, GA21, MON810, T25) and a screening quantitative method targeting Cauliflower Mosaic Virus 35S promoter (P35S) and GA21 have been adopted as Japanese standard analytical methods ${ }^{* 5}$. MIR162 is an insect-resistant GM maize event and its commercial utilization in Japan was approved in $2010^{* 6}$. In MIR162, a single copy of the modified vip $3 A$ gene from Bacillus thuringiensis strain $\mathrm{AB} 88$ and a selectable marker gene

*3 Consumer Affairs Agency, Government of Japan (2011) http://www.caa.go.jp/foods/pdf/syokuhin737.pdf

*4 Notification No. 14932 (Apr. 1, 2007); Ministry of Agriculture, Forestry and Fisheries of Japan (2007) http://www. caa.go.jp/jas/hyoji/pdf/tuuti_a.pdf

*5 Notification No. 201 (Nov. 16, 2012); Consumer Affairs Agency, Government of Japan (2012) http://www.caa.go.jp/ foods/pdf/syokuhin960.pdf

${ }^{* 6}$ List of products that have undergone safety assessment and been announced in the Official Gazette (Apr. 10, 2014) of the Department of Food Safety, Ministry of Health, Labour and Welfare of Japan; http://www.mhlw.go.jp/english/ topics/food/pdf/sec01-2.pdf 


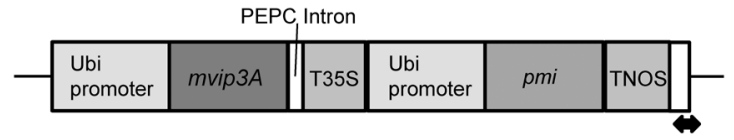

Fig. 1. A schematic diagram of the target position in MIR162

The event-specific target sequence is designed at the $3^{\prime}$-flanking region between the exogenous insert and host maize DNA.

mannose-6-phosphate isomerase ( $p m i$ ) from Escherichia coli have been introduced without the P35S region (Fig. 1). Thus, a specific detection method for MIR162 is required.

In the present study, we developed a new quantitative method for MIR162 with an event-specific PCR method, and then validated the method in an interlaboratory study.

\section{Materials and Methods}

\section{Plant materials}

For maize and soybean, MON810, MON863, MON88017, NK603, MON89788, and RRS were kindly provided by Monsanto Company (St. Louis, MO, USA). 3272, Bt11, Event176, GA21, MIR162, and MIR604 were kindly provided by Syngenta Seeds AG (Basel, Switzerland); and TC1507 and DAS59122 were kindly provided by Pioneer Hi-Bred International (Johnston, IA, USA). A2704-12 was kindly provided by its developer and T25 was directly imported from the USA. QC9651 maize obtained from Quality Technology International, Inc. (Elgin, IL, USA) was used as non-GM maize. Seeds of rice (Oryza sativa) variety Kinuhikari, wheat (Triticum aestivum) variety Haruyutaka, and barley (Hordeum vulgare) variety Harrington in Japan were obtained from the market.

\section{Oligonucleotide primers and probes}

For the specific detection of MIR162, a pair of primers (5'-GCGCGGTGTCATCTATGTTACTAG-3' and 5'-TGC CTTATCTGTTGCCTTCAGA-3') and a fluorescent dyelabeled probe (5'-TCTAGACAATTCAGTACATTAAAA ACGTCCGCCA-3') were used. The sequences of these primers and probe were taken from a report by the Institute for Health and Consumer Protection, the European Commission's Joint Research Centre (JRC) (Ispra, Italy)*7. Maize starch synthase IIb (SSIIb) gene was used as a maize-specific endogenous reference DNA for quantitative analysis. For the specific detection of SSIIb, two primers (5'-CCAATCCTTTGACATCTGCTCC- $3^{\prime}$ and 5'-GATCAG CTTTGGGTCCGGA-3') and a fluorescent dye-labeled probe ( $5^{\prime}$-AGCAAAGTCAGAGCGCTGCAATGCA-3') were used $^{3)}$. The oligonucleotide primers and TaqMan ${ }^{\circledR}$ probes were synthesized by FASMAC Co., Ltd. (Kanagawa, Japan) and Life Technologies (Carlsbad, CA, USA), respec-

\footnotetext{
${ }^{* 7}$ Event-specific method for the quantification of maize MIR162 using real-time PCR; http://gmo-crl.jrc.ec.europa. eu/summaries/MIR162_validated_Method.pdf
}

tively. The oligonucleotide probes were labeled with 6-carboxyfluorescein (FAM) at the $5^{\prime}$ ends and 6-carboxytetramethylrhodamin (TAMRA) at the $3^{\prime}$ ends.

\section{Preparation of the calibrant plasmid}

Target sequence fragments from MIR162 and the endogenous maize SSIIb gene were synthesized as a single oligonucleotide in tandem and inserted into a pUC19 derived vector. The constructed plasmid was purified by equilibrium centrifugation in $\mathrm{CsCl}$ gradient. The concentration of the linearized DNA was first measured in terms of ultraviolet (UV) absorbance with a spectrophotometer, DU800 (Beckman Coulter, Brea, CA, USA), and converted to molar concentration. The DNA was then diluted to theoretical concentrations of 20, 125, 1,500, 20,000 , and 250,000 copies per $2.5 \mu \mathrm{L}$ with $5 \mathrm{ng} / \mu \mathrm{L}$ of ColE1 DNA (NIPPON GENE, Tokyo, Japan) solution.

\section{Preparation of test samples and DNA extraction}

To evaluate the quantitative method, we used five mixing levels of test materials containing $0 \%, 0.50 \%, 1.0 \%$, $5.0 \%$, and $10.0 \%$ of MIR162. To prepare the mixed samples, GM and non-GM seeds were separately ground, and then mixed on a weight-basis as described previously ${ }^{4), 5)}$. Genomic DNA was extracted from those ground materials using a DNeasy Plant Maxi Kit (Qiagen, Hilden, Germany). The concentrations and qualities of the extracted DNA solutions were evaluated by measuring UV absorbance with a spectrophotometer, ND-100 (NanoDrop Technologies, Wilmington, DE, USA). Maize genomic DNA solutions were adjusted to concentrations of 10 and $20 \mathrm{ng} / \mu \mathrm{L}$ for conventional and real-time PCR analyses, respectively.

\section{Conventional PCR analysis}

Conventional PCR using a thermal cycler, GeneAmp PCR system 9700 (Life Technologies), and agarose gel electrophoresis were performed as described previously by Kuribara et al. ${ }^{6)}$. Twenty-five ng of sample DNA, $200 \mu \mathrm{M}$ deoxyribonucleotide triphosphate (dNTP), $1.5 \mathrm{mM} \mathrm{MgCl}, 0.625 \mathrm{U}$ of AmpliTaq Gold polymerase (Life Technologies) and $0.5 \mu \mathrm{M}$ each primer were added to $25 \mu \mathrm{L}$, of reaction solution. The thermal cycling condition was set as $10 \mathrm{~min}$ at $95^{\circ} \mathrm{C}$ and 40 cycles of $30 \mathrm{sec}$ at $95^{\circ} \mathrm{C}, 30 \mathrm{sec}$ at $60^{\circ} \mathrm{C}$ and $30 \mathrm{sec}$ at $72^{\circ} \mathrm{C}$, followed by a final extension at $72^{\circ} \mathrm{C}$ for $7 \mathrm{~min}$. Five microliters of PCR products were electrophoresed on $3.0 \%$ agarose gel supplemented with $0.5 \mu \mathrm{g} / \mathrm{mL}$ of ethidium bromide in Trisacetate-EDTA (TAE) buffer.

\section{Real-time PCR analysis}

TaqMan $^{\circledR}$ real-time PCR assays were carried out using two real-time PCR instruments, the Applied Biosystems 7900HT (ABI7900) and the Applied Biosystems 7500 (ABI7500) (Life Technologies), with $25 \mu \mathrm{L}$ final volume reactions consisting of $50 \mathrm{ng}$ of sample DNA, $12.5 \mu \mathrm{L}$ Universal Master Mix (Life Technologies), $0.5 \mu \mathrm{M}$ primer pairs, and $0.2 \mu \mathrm{M}$ probe. The thermal cycling condition was set as $2 \mathrm{~min}$ at $50{ }^{\circ} \mathrm{C}, 10 \mathrm{~min}$ at $95^{\circ} \mathrm{C}$ and $45 \mathrm{cy}$ - 
cles of $30 \mathrm{sec}$ at $95^{\circ} \mathrm{C}, 1 \mathrm{~min}$ at $59^{\circ} \mathrm{C}$. Each sample was measured in triplicate on the reaction plate.

\section{Interlaboratory study}

An interlaboratory study was performed with the ABI7900 and ABI7500 independently and consisted of 2 separate stages, a measurement of the conversion factor (Cf) value and a blind test. All measurements were conducted by 10 laboratories for the ABI7900 and 3 laboratories for the ABI7500. Experimental protocols were provided by the National Food Research Institute (NFRI). Quantitative real-time PCR was performed with primers, probes, the Universal Master Mix, and the blind DNA solutions supplied by the NFRI.

The first stage was the experimental determination of the $\mathrm{Cf}$ value, which is the ratio of the copy number of recombinant DNA (rDNA) to the taxon-specific sequence in the GM plant genome. To calculate the $\mathrm{Cf}$ value for MIR162, we extracted genomic DNA from genuine GM seeds and determined the copy numbers of rDNA and taxon-specific sequences. The measurement was conducted twice at each laboratory. The Cf value for each real-time PCR instrument was separately determined as the mean of the obtained values.

A blind test was carried out as the second stage. Blind samples designed as blind duplicates of the maize genomic DNAs extracted from 5 different concentrations of MIR162 (0\%, $0.50 \%, 1.0 \%, 5.0 \%$, and $10.0 \%)$ were provided to the participants. All participants were requested to submit the data of their real-time PCR analyses. All submitted data were analyzed by Cochran's test ${ }^{7)}$ and Grubbs' test ${ }^{8,9)}$ to reject outlier laboratories.

\section{Results and Discussion}

\section{PCR specificity for MIR162}

For the specific detection of MIR162, an event-specific segment was amplified at the $3^{\prime}$ flanking region between the native maize genomic DNA and rDNA (Fig. 1). First, the specificity of the primer set was confirmed by a conventional PCR method. The expected 92-bp product was detected only from genomic DNA solution of MIR162, but not from non-GM maize, other GM maize events, GM soybean events, rice, wheat, barley, and the no-template control (Fig. 2 A). Next, the specificity of the primer and probe set was confirmed by a real-time PCR analysis. The expected amplification curves were observed using the genomic DNA of MIR162 but not from those of the other samples (Fig. 2 B).

\section{Determination of the Cf value for MIR162}

The Cf value is required to convert the copy number ratio of rDNA to taxon-specific DNA into weight-based GMO content. To determine the experimental Cf value for MIR162, we measured the copy numbers of $S S I I b$ and MIR162 in the extracted DNA from MIR162 seeds. The $\mathrm{Cf}$ value was determined from the results of 10 laboratories for the $\mathrm{ABI} 7900$ and the measurement was repeated twice. All submitted data were subjected to Cochran's test $(p<0.025)$ and Grubb's test $(p<0.025)$ to eliminate outlier laboratories with extreme variation and extreme average level, respectively, according to the harmonized guidelines of $\mathrm{AOAC}^{10)}$. One Cochran outlier

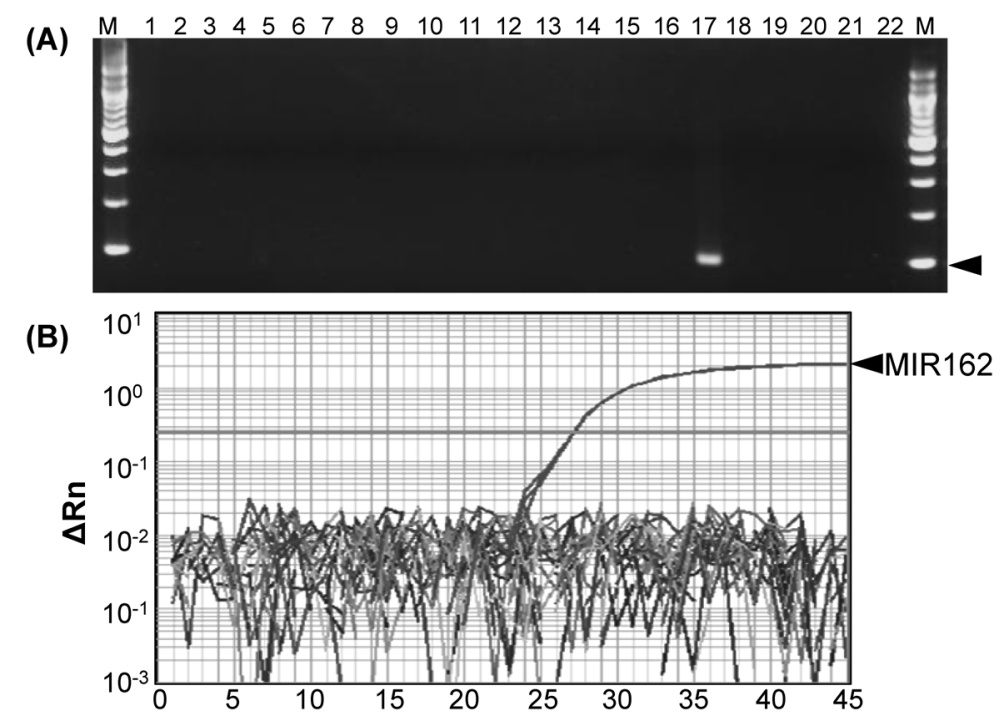

Fig. 2. Specificity test for the MIR162-specific PCR

(A) Conventional PCR analysis. This agarose gel (3.0\%) electrophoretogram of the amplified PCR product corresponds to the $92 \mathrm{bp}$ of MIR162. Arrowhead indicates the expected amplified product. Lanes 1-11, eleven GM maize events, namely, NK603, Event176, T25, GA21, MON810, TC1507, Bt11, MIR604, MON88017, DAS59122, and MON863, respectively; lanes 12 and 13, non-GM maize and non-GM soy, respectively; lanes 14-16, three GM soybean events, namely, RRS, MON89788, and A2704-12, respectively; lanes 17 and 18, GM maize events, MIR162 and 3272, respectively; lanes 19-22, rice, wheat, barley, and no template, respectively. Lane M shows 100 bp ladder size markers. (B) Real-time PCR analysis. The same set of plant genomic DNAs described in (A) were used as templates. The realtime PCR analysis was performed in one reaction plate, and the resultant amplification plot is shown. This analysis was performed with the 7900HT. Only the MIR162 genomic DNA gave ideal PCR amplification. 
was detected. After removing the outlier, further statistical analyses were conducted. The $\mathrm{Cf}$ value was finally determined as the mean of values measured by the remaining laboratories. The determined $\mathrm{Cf}$ value was 0.697 (Table 1). We also determined Cf value of 0.635 for MIR162 using ABI 7500 as a reference value from the results of 3 laboratories (Table 1 ).

\section{Evaluation of the PCR quantitative method by interlabo-} ratory study

We performed an interlaboratory evaluation of the developed quantitative method as a blind test performed by 10 laboratories using the ABI7900. All participants received primers, probes and blind samples of DNA solutions consisting of five different concentrations of MIR162, and each measurement was performed twice. The blank sample ( $0 \%$ of MIR162) was used to determine outlier laboratories, but no laboratory was eliminated. The submitted data of 4 individual concentration samples were subjected to Cochran's test and Grubbs' test, but no outliers were found. $R^{2}$ value is the coefficient of correlation, which is used to analyze a standard curve obtained by linear regression analysis. We have an acceptance criteria that $R^{2}$ values must be $>0.990^{6)}$. In Europe, minimum performance requirements for analytical methods of GMO testing have been defined by the

Table 1. Experimental conversion factor for MIR162

\begin{tabular}{ccccccc}
\hline \hline & 7900 & & & \multicolumn{3}{c}{7500} \\
\cline { 1 - 2 } \cline { 5 - 6 } Mean & SD & RSD & & Mean & SD & RSD \\
\hline 0.697 & 0.060 & 8.67 & & 0.635 & 0.019 & 2.97 \\
\hline
\end{tabular}

SD: Standard deviation

RSD: Relative standard deviation
European Network of GMO Laboratories (ENGL), and $R^{2}$ values should be $\geq 0.98$ in the criteria ${ }^{* 8}$. We listed the $R^{2}$ values for the standard curves using $20,125,1,500$, 20,000 , and 250,000 copies of standard plasmids from each participant in the two quantitative analyses as blind duplicates (Table 2), and all of the obtained $R^{2}$ values from both SSIIb and MIR162 met the acceptance level. Trueness and precision were determined as previously described ${ }^{3)-5)}$. The mean, bias (mean-value, \%), repeatability of relative standard deviation (RSDr), and reproducibility of RSD (RSDR) blind samples were measured (Table 3). The determined bias, RSDr, and RSDR for $\mathrm{ABI} 7900$ ranged from $12.3 \%$ to $21.9 \%$, from $8.4 \%$ to $17.0 \%$, and from $9.6 \%$ to $17.0 \%$, respectively. RSDR values indicate the variance of the quantification of each concentration, and the variance of the $0.5 \%$ sample which was the lowest concentration, was $11.3 \%$. The obtained bias, RSDr, and RSDR here were similar to or within a narrower range than those for previously reported GMO events ${ }^{3-5)}$. Data below 20 copies were extrapolated with a standard curve in our methods because there was no calibrant below 20 copies. For quantitative methods the limit of detection (LOD) is the amount of analyte at which the analytical method detects the presence of the analyte at least $95 \%$ of the time $(\leq 5 \% \text { false negative results })^{* 9}$. Twenty copies of standard plasmid were detected by all participants in the two quantitative analyses, indicating that the ratio of the detection was 20/20 and the LOD was 20 copies. All measured copies of $0.50 \%$ samples were over 20 copies (Table 3). Therefore, we estimate that the limit of quantitation (LOQ) for MIR162 is $0.50 \%$ in this method.

In conclusion, we developed a specific quantification method for GM maize MIR162, using the same primers

Table 2. $R^{2}$ values of the standard curves drawn by each participant

\begin{tabular}{ccccccccccc}
\hline \hline Participant & A & B & C & D & E & F & G & H & I & J \\
\hline SSIIb & 0.999 & 0.999 & 0.998 & 0.998 & 0.999 & 0.999 & 0.999 & 0.997 & 0.998 & 0.999 \\
MIR162 & 0.998 & 0.998 & 0.999 & 0.999 & 0.997 & 1.000 & 0.997 & 0.996 & 0.998 & 0.997 \\
SSIIb & 1.000 & 0.999 & 0.999 & 0.999 & 0.998 & 0.999 & 0.999 & 0.999 & 1.000 & 0.999 \\
MIR162 & 0.996 & 0.999 & 0.999 & 1.000 & 1.000 & 0.996 & 0.997 & 0.998 & 0.998 & 0.997 \\
\hline
\end{tabular}

Table 3. Summary of accuracy and precision statistics for real-time PCR with ABI PRISM 7900HT

\begin{tabular}{|c|c|c|c|c|c|c|c|c|}
\hline \multirow{3}{*}{$\%(\mathrm{w} / \mathrm{w})$} & \multirow{3}{*}{ Retained labs } & \multicolumn{2}{|c|}{ Trueness } & \multicolumn{4}{|c|}{ Precision } & \multirow{3}{*}{$\begin{array}{c}\text { LOD } \\
(20 \text { copies }) \\
\text { Below LOD }\end{array}$} \\
\hline & & \multirow{2}{*}{$\frac{\text { Means }}{\text { GMO Amount, \% }}$} & \multirow{2}{*}{$\frac{\text { Bias }}{\text { True value, \% }}$} & \multicolumn{2}{|c|}{ Repeatability } & \multicolumn{2}{|c|}{ Reproducibility } & \\
\hline & & & & $\mathrm{Sr}$ & RSDr, \% & $\mathrm{SR}$ & RSDR, \% & \\
\hline 0.5 & 10 & 0.55 & 10 & 0.062 & $11.3 \mathrm{a}$ & 0.057 & $11.3 \mathrm{a}$ & $0 / 20$ \\
\hline 1 & 10 & 1.177 & 15 & 0.202 & $17.2 \mathrm{a}$ & 0.194 & $17.2 \mathrm{a}$ & $0 / 20$ \\
\hline 5 & 10 & 6.035 & 20.7 & 0.567 & 9.4 & 0.584 & 9.7 & $0 / 20$ \\
\hline 10 & 10 & 12.051 & 20.5 & 1.026 & 8.5 & 1.2 & 10 & $0 / 20$ \\
\hline
\end{tabular}

${ }^{a}$ When $\mathrm{RSDr}>\mathrm{RSDR}$, RSDR was considered to have the same value as RSDr.

*8 Definition of Minimum Performance Requirements for Analytical Methods of GMO Testing, European Network of GMO Laboratories (ENGL) http://gmo-crl.jrc.ec.europa.eu/ doc/Min_Perf_Requirements_Analytical_methods.pdf
*9 Guidelines on performance criteria and validation of methods for detection, identification and quantification of specific DNA sequences and specific proteins in foods* CAC/GL 74-2010 
and probe sequences for the MIR162 transgene as those described in the method developed by the trait develop$\mathrm{er}^{* 7}$. The experimentally determined Cf value was 0.697 for the ABI7900. The LOQ, trueness, and precision of this method were at the same level or better than those reported in previous studies ${ }^{3-5)}$. Therefore, we conclude that the developed method would be applicable for the detection and quantification of MIR162 to monitor the validity of the food labeling system in Japan.

\section{Acknowledgements}

The authors would like to thank the following collaborators for their participation in this study:

FASMAC, Kanagawa, Japan

Food and Agricultural Materials Inspection Center, Saitama, Japan

Japan Food Research Laboratories, Tokyo, Japan

Japan Frozen Foods Inspection Corporation, Kanagawa, Japan

Japan Grain Inspection Association, Tokyo, Japan

Life Technologies Japan, Tokyo, Japan

National Center for Seed and Seedlings, Ibaraki, Japan

National Food Research Institute, Ibaraki, Japan

National Institute of Health Sciences, Tokyo, Japan

Yokohama Plant Protection Station, Kanagawa, Japan

We would like to thank Shigehiro Naito (National Food Research Institute, National Agriculture and Food Research Organization, Ibaraki, Japan) for his excellent suggestions on the statistical aspects of this study. This work was supported by Research Projects ("Assurance of Safe Use of Genetically Modified Organisms" and "Research Project for Genomics for Agricultural Innovation GAM-210”) funded by the Ministry of Agriculture, Forestry, and Fisheries of Japan, a Grant from the Ministry of Health, Labour and Welfare of Japan, and a Grant from the Consumer Affairs Agency, Government of Japan.

\section{References}

1) James, C. Global Status of Commercialized Biotech/GM Crops: 2012. ISAAA Brief 44. Ithaca, ISAAA, 2012 (ISBN 978-1-892456-53-2)

2) Hino, A. Safety assessment and public concerns for genetically modified food products: The Japanese experience. Toxicol. Pathol., 30, 126-128 (2002).

3) Kodama, T., Kuribara, H., Minegishi, Y., Futo, S., Watai, M., Sawada, C., Watanabe, T., Akiyama, H., Maitani, T., Teshima, R., Furui, S., Hino, A., Kitta, K. Evaluation of modified PCR quantitation of genetically modified maize and soybean using reference molecules: interlaboratory study. J. AOAC Int., 92, 223-233 (2009).

4) Takabatake, R., Akiyama, H., Sakata, K., Onishi, M., Koiwa, T., Futo, S., Minegishi, Y., Teshima, R., Mano, J., Furui, S., Kitta, K. Development and evaluation of event-specific quantitative PCR method for genetically modified soybean A2704-12. Food Hyg. Saf. Sci., 52, 100107 (2011).

5) Mano, J., Masubuchi, T., Hatano, S., Futo, S., Koiwa, T., Minegishi, Y., Noguchi, A., Kondo, K., Akiyama, H., Teshima, R., Kurashima, T., Takabatake, R., Kitta, K. Development and validation of event-specific quantitative PCR method for genetically modified maize LY038. Food Hyg. Saf. Sci., 54, 25-30 (2013).

6) Kuribara, H., Shindo, Y., Matsuoka, T., Takubo, K., Futo, S., Aoki, N., Hirao, T., Akiyama, H., Goda, Y., Toyoda, M., Hino, A. Novel reference molecules for quantification of genetically modified maize and soybean. J. AOAC Int., 85, 1077-1089 (2002).

7) Cochran, W. G. The distribution of the largest of a set of estimated variances as a fraction of their total, Annals of Eugenics, 11, 47-52 (1941).

8) Grubbs, F. E. Samples criteria for testing outlying observations, Ann. Math. Statist. Assn., 21, 27-58 (1950).

9) Grubbs, F. E. Procedures for detecting outlying observations in samples, Technometrics, 11, 1-21 (1969).

10) Appendix D.: Guidelines for Collaborative Study Procedures to Validate Characteristics of a Method of Analysis. In Official Methods of Analysis of AOAC Int., 17th ed., Volume II, Gaithersburg, MD, USA. 